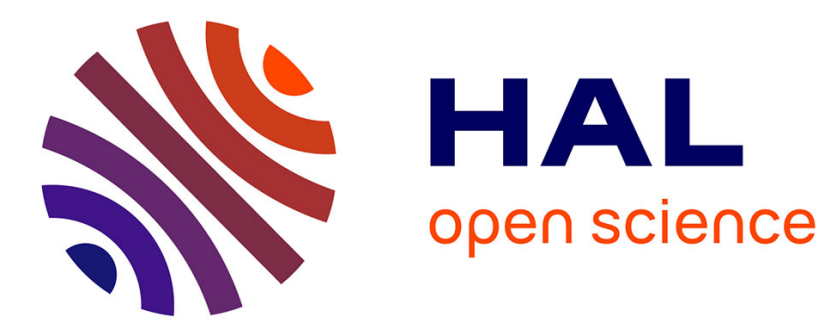

\title{
Power Efficient Low Complexity Precoding for Massive MIMO Systems
}

\author{
Houssem Sifaou, Abla Kammoun, Luca Sanguinetti, Mérouane Debbah, \\ Mohamed-Slim Alouini
}

\section{- To cite this version:}

Houssem Sifaou, Abla Kammoun, Luca Sanguinetti, Mérouane Debbah, Mohamed-Slim Alouini. Power Efficient Low Complexity Precoding for Massive MIMO Systems. 2014 IEEE Global Conference on Signal and Information Processing (GlobalSIP), Dec 2014, Atlanta, United States. 10.1109/globalsip.2014.7032197 . hal-01098935

\section{HAL Id: hal-01098935 \\ https://hal.science/hal-01098935}

Submitted on 17 Jan 2015

HAL is a multi-disciplinary open access archive for the deposit and dissemination of scientific research documents, whether they are published or not. The documents may come from teaching and research institutions in France or abroad, or from public or private research centers.
L'archive ouverte pluridisciplinaire HAL, est destinée au dépôt et à la diffusion de documents scientifiques de niveau recherche, publiés ou non, émanant des établissements d'enseignement et de recherche français ou étrangers, des laboratoires publics ou privés. 


\title{
Power Efficient Low Complexity Precoding for Massive MIMO Systems
}

\author{
Houssem Sifaou $^{\dagger \ddagger}$, Abla Kammoun ${ }^{\dagger}$, Luca Sanguinetti ${ }^{\star \diamond}$, Mérouane Debbah ${ }^{\diamond *}$, Mohamed-Slim Alouini ${ }^{\dagger}$ \\ $\dagger$ Electrical Engineering Department, King Abdullah University of Science and Technology, Thuwal, Saudi Arabia \\ $\ddagger$ Signals and Systems Department, Ecole Polytechnique de Tunisie, La Marsa, Tunisia \\ $\star$ Dipartimento di Ingegneria dell'Informazione, University of Pisa, Pisa, Italy \\ $\diamond$ Ecole supérieure d'électricité (Supélec), Gif-sur-Yvette, France \\ * Mathematical and Algorithmic Sciences Lab, Huawei France R\&D, Paris, France.
}

\begin{abstract}
This work aims at designing a low-complexity precoding technique in the downlink of a large-scale multiple-input multiple-output (MIMO) system in which the base station (BS) is equipped with $M$ antennas to serve $K$ single-antenna user equipments. This is motivated by the high computational complexity required by the widely used zero-forcing or regularized zero-forcing precoding techniques, especially when $K$ grows large. To reduce the computational burden, we adopt a precoding technique based on truncated polynomial expansion (TPE) and make use of the asymptotic analysis to compute the deterministic equivalents of its corresponding signal-to-interference-plus-noise ratios (SINRs) and transmit power. The asymptotic analysis is conducted in the regime in which $M$ and $K$ tend to infinity with the same pace under the assumption that imperfect channel state information is available at the BS. The results are then used to compute the TPE weights that minimize the asymptotic transmit power while meeting a set of target SINR constraints. Numerical simulations are used to validate the theoretical analysis.
\end{abstract}

\section{INTRODUCTION}

Large-scale multiple-input multiple-output (MIMO) systems (also known as massive MIMO systems) are considered as one of the most promising technology for next generation wireless communication systems [1]. The use of an excess number $M$ of antennas at the base station (BS) provides the system with many degrees of freedom to substantially reduce multi-user interference [1]. In downlink transmissions, this is usually accomplished using linear precoding techniques whose complexity, however, increase substantially when the number $K$ of user equipments (UEs) grows large. Among the different techniques, the most popular ones rely on zero-forcing (ZF) or regularized zero-forcing (RZF) approaches [2]-[8]. In [6], it is proved that RZF precoder has the same performance of the optimal one [9] if the regularization parameter is properly designed and the same signal-to-interference-plus-noise ratio (SINR) constraints are imposed for all UEs. Unfortunately, both ZF and RZF involve the computation of the inverse of a matrix, which poses serious challenges towards its practical implementation especially when $K$ grows large and the propagation channel changes relatively fast in time. A possible solution to overcome this issue is proposed in [4] in which the authors make use of the truncated polynomial expansion (TPE) technique to reduce the computational complexity required by matrix inversion. In [4], the polynomial coefficients are designed so as to maximize the achievable rate subject to a power constraint. It is worth observing that TPE techniques have been also applied in other contexts such as channel estimation [10] and multi-user detection [11], [12]. In all cases, the main advantage of TPE is a substantial complexity reduction with respect to linear processing techniques (see [4] for more details on this).

Research reported in this publication was supported by the King Abdullah University of Science and Technology (KAUST). It has also received funding from ERC Grant 305123 MORE. L. Sanguinetti is funded by the People Programme (Marie Curie Actions) FP7 PIEF-GA-2012-330731 "Dense4Green".
In this work, we aim at designing the TPE precoding scheme presented in [4] to minimize the power minimization while satisfying SINR constraints under the assumption that imperfect channel state information (CSI) is available at the BS. The analysis is conducted in the asymptotic regime in which $M$ and $K$ tend to infinity with the same pace. This allows us to simplify the design methodology since in the asymptotic regime performance metrics converge to deterministic quantities, which can be well-approximated using tools borrowed from random matrix theory [2], [4], [5]. These tools are used henceforth to derive deterministic equivalents of SINRs and transmit power. The latter are eventually used to determine the optimal TPE coefficients minimizing the transmit power under the set of SINR constraints.

\section{SYSTEM DESCRIPTION AND SIGNAL MODEL}

We consider a single-cell massive MIMO system in which the BS is equipped with $M$ antennas and serves $K$ single antenna UEs, which are randomly selected from a larger set. The location of UE $k$ is characterized by its distance $d_{k}$ from the BS (located in the centre of the cell for simplicity). We denote by $\beta_{k}$ the average large-scale channel attenuation due to pathloss and shadowing at distance $d_{k}$ and assume that it is the same for all BS antennas 1 The channel vector $\mathbf{h}_{k}$ for UE $k$ is modeled as:

$$
\mathbf{h}_{k}=\sqrt{\beta_{k}} \mathbf{z}_{k}
$$

where $\mathbf{z}_{k} \sim \mathcal{C N}\left(\mathbf{0}_{M \times 1}, \boldsymbol{\Phi}\right)$ and $\boldsymbol{\Phi} \in \mathbb{C}^{M \times M}$ stands for the channel covariance matrix, which is assumed to be the same for all UEs. We assume that imperfect channel state information (CSI) is available at the BS. This is modelled for UE $k$ by [2]:

$\widehat{\mathbf{h}}_{k}=\sqrt{\beta_{k}} \widehat{\mathbf{z}}_{k}=\sqrt{\beta_{k}}\left(\sqrt{1-\tau^{2}} \mathbf{z}_{k}+\tau \mathbf{v}_{k}\right)=\sqrt{1-\tau^{2}} \mathbf{h}_{k}+\sqrt{\beta_{k}} \tau \mathbf{n}_{k}$

where $\mathbf{v}_{k}, \widehat{\mathbf{z}}_{k} \sim \mathcal{C N}\left(\mathbf{0}_{M \times 1}, \boldsymbol{\Phi}\right)$ and $\mathbf{n}_{k}=\boldsymbol{\Phi}^{\frac{1}{2}} \mathbf{v}_{k} \sim \mathcal{C N}\left(\mathbf{0}_{M \times 1}, \boldsymbol{\Phi}\right)$ stands for additive Gaussian noise. The scalar parameter $\tau \in[0,1]$ indicates the quality of CSI: $\tau=0$ corresponds to perfect CSI whereas $\tau=1$ corresponds to statistical channel knowledge. For notational convenience, we call $\widehat{\mathbf{H}}=\left[\widehat{\mathbf{h}}_{1}, \cdots, \widehat{\mathbf{h}}_{K}\right]$, which can be written in matrix form as

$$
\widehat{\mathbf{H}}=\widehat{\mathbf{Z}} \mathcal{B}^{\frac{1}{2}}
$$

where $\mathcal{B}=\operatorname{diag}\left(\beta_{1}, \cdots, \beta_{K}\right)$ and $\widehat{\mathbf{Z}}=\left[\widehat{\mathbf{z}}_{1}, \cdots, \widehat{\mathbf{z}}_{K}\right]$. We denote by $\mathbf{G}=\left[\mathbf{g}_{1}, \cdots, \mathbf{g}_{K}\right]$ the precoding matrix and call $\mathbf{s}=\left[s_{1}, \cdots, s_{K}\right]$ the vector containing all UE data symbols. Consequently, the received signal at UE $k$ can be expressed as:

$$
y_{k}=\mathbf{h}_{k}^{\mathrm{H}} \mathbf{g}_{k} s_{k}+\sum_{n=1, n \neq k}^{K} \mathbf{h}_{k}^{\mathrm{H}} \mathbf{g}_{n} s_{n}+n_{k} .
$$

${ }^{1}$ This is reasonable since the distances between UEs and BS are much larger than the distance between the BS antennas. 
Let $\mathbf{G}_{k}$ be the matrix $\mathbf{G}$ with column $\mathbf{g}_{k}$ removed. Then, the SINR at UE $k$ can be expressed as:

$$
\operatorname{SINR}_{k}=\frac{\mathbf{h}_{k}^{\mathrm{H}} \mathbf{g}_{k} \mathbf{g}_{k}^{\mathrm{H}} \mathbf{h}_{k}}{\mathbf{h}_{k}^{\mathrm{H}} \mathbf{G} \mathbf{G}^{\mathrm{H}} \mathbf{h}_{k}-\mathbf{h}_{k}^{\mathrm{H}} \mathbf{g}_{k} \mathbf{g}_{k}^{\mathrm{H}} \mathbf{h}_{k}+\sigma^{2}} .
$$

Using the truncated polynomial approximation presented in [4], the precoding matrix $\mathbf{G}$ is designed as follows:

$$
\mathbf{G}_{\mathrm{TPE}}=\sum_{k=0}^{J-1} w_{k}\left(\frac{1}{K} \widehat{\mathbf{H}} \mathcal{B}^{-1} \widehat{\mathbf{H}}^{\mathrm{H}}\right)^{k} \frac{\widehat{\mathbf{H}}}{\sqrt{K}} \mathbf{P}^{\frac{1}{2}}
$$

where $\mathbf{P}=\operatorname{diag}\left(p_{1}, \cdots, p_{K}\right)$ with $p_{k}$ being the power allocated to $\mathrm{UE} k, \mathbf{w}=\left[w_{0}, \cdots, w_{J-1}\right]^{T}$ is a design vector and $J$ is the polynomial degree, which allows (if properly selected) to obtain different precoding techniques. Observe that in designing $\mathbf{G}_{\mathrm{TPE}}$ we have assumed knowledge of the large-scale fading matrix $\mathcal{B}$. This is a reasonable assumption since the large-scale fading attenuations change slowly with time (relative to the small-scale fading) and thus can be accurately estimated at the BS. Plugging (6) into 5 yields

$$
\operatorname{SINR}_{k}=\frac{K p_{k} \mathbf{w}^{\mathrm{T}} \mathbf{A}_{k} \mathbf{w}}{\mathbf{w}^{\mathrm{T}} \mathbf{B}_{k} \mathbf{w}+\sigma^{2}}
$$

where the matrices $\mathbf{A}_{k}$ and $\mathbf{B}_{k}$ are defined as:

$\left[\mathbf{A}_{k}\right]_{\ell, m}=\frac{\beta_{k}^{2}}{K^{2}} \mathbf{z}_{k}^{\mathrm{H}}\left(\frac{1}{K} \widehat{\mathbf{H}} \mathcal{B}^{-1} \widehat{\mathbf{H}}^{\mathrm{H}}\right)^{\ell} \widehat{\mathbf{z}}_{k} \widehat{\mathbf{z}}_{k}^{\mathrm{H}}\left(\frac{1}{K} \widehat{\mathbf{H}} \mathcal{B}^{-1} \widehat{\mathbf{H}}^{\mathrm{H}}\right)^{m} \mathbf{z}_{k}$

$\left[\mathbf{B}_{k}\right]_{\ell, m}=\frac{\beta_{k}}{K} \mathbf{z}_{k}^{\mathrm{H}}\left(\frac{1}{K} \widehat{\mathbf{H}} \mathcal{B}^{-1} \widehat{\mathbf{H}}^{\mathrm{H}}\right)^{\ell} \widehat{\mathbf{H}} \mathbf{P} \widehat{\mathbf{H}}^{\mathrm{H}}\left(\frac{1}{K} \widehat{\mathbf{H}} \mathcal{B}^{-1} \widehat{\mathbf{H}}^{\mathrm{H}}\right)^{m} \mathbf{z}_{k}-\left[\mathbf{A}_{k}\right]_{\ell, m}$. and

The corresponding transmit power turns out to be [4]

$$
P_{\mathrm{TPE}}=\operatorname{tr}\left(\mathbf{G}_{\mathrm{TPE}} \mathbf{G}_{\mathrm{TPE}}^{\mathrm{H}}\right)=\mathbf{w}^{\mathrm{T}} \mathbf{E} \mathbf{w}
$$

where the $(\ell, m)$-th element of the matrix $\mathbf{E}$ is computed as:

$$
[\mathbf{E}]_{\ell, m}=\frac{1}{K} \operatorname{tr}\left(\left(\frac{\widehat{\mathbf{H}} \mathcal{B}^{-1} \widehat{\mathbf{H}}^{\mathrm{H}}}{K}\right)^{\ell} \widehat{\mathbf{H}} \mathbf{P} \widehat{\mathbf{H}}^{\mathrm{H}}\left(\frac{\widehat{\mathbf{H}} \mathcal{B}^{-1} \widehat{\mathbf{H}}^{\mathrm{H}}}{K}\right)^{m}\right) \text {. }
$$

The large system analysis is used to compute the asymptotic expressions or deterministic equivalents of the SINRs and transmit power defined above.

\section{ASYMPTOTIC ANALYSIS OF TPE PRECODING}

From (7) and (9), it follows that the deterministic equivalents of SINR $_{k}$ and $P_{\text {TPE }}$ require to find the asymptotic approximations of the matrices $\mathbf{A}_{k}, \mathbf{B}_{k}$ and $\mathbf{E}$. To this end, we observe that $\mathbf{A}_{k}, \mathbf{B}_{k}, \mathbf{E}$ are respectively (up to a scaling factor) the higher order derivatives of

$$
\begin{aligned}
& X_{k}(t, u)=\frac{1}{K^{2}} \mathbf{z}_{k}^{\mathrm{H}} \mathbf{Q}(t) \widehat{\mathbf{z}}_{k} \widehat{\mathbf{z}}_{k}^{\mathrm{H}} \mathbf{Q}(u) \mathbf{z}_{k} \\
& Z_{k}(t, u)=\frac{1}{K} \mathbf{z}_{k}^{\mathrm{H}} \mathbf{Q}(t) \widehat{\mathbf{H}} \mathbf{P} \widehat{\mathbf{H}}^{\mathrm{H}} \mathbf{Q}(u) \mathbf{z}_{k} \\
& Y(t, u)=\frac{1}{K} \operatorname{tr}\left(\mathbf{Q}(t) \widehat{\mathbf{H}} \mathbf{P} \widehat{\mathbf{H}}^{\mathrm{H}} \mathbf{Q}(u)\right)
\end{aligned}
$$

evaluated at $t=u=0$. In particular, it turns out that:

$$
\begin{aligned}
{\left[\mathbf{A}_{k}\right]_{\ell, m} } & =\frac{\beta_{k}^{2}(-1)^{\ell+m}}{\ell ! m !} X_{k}^{(\ell, m)} \\
{\left[\mathbf{B}_{k}\right]_{\ell, m} } & =\frac{\beta_{k}(-1)^{\ell+m}}{\ell ! m !}\left(Z_{k}^{(\ell, m)}-K p_{k} X_{k}^{(\ell, m)}\right) \\
{[\mathbf{E}]_{\ell, m} } & =\frac{(-1)^{\ell+m}}{\ell ! m !} Y^{(\ell, m)}
\end{aligned}
$$

where $X_{k}^{(\ell, m)}, Z_{k}^{(\ell, m)}$ and $Y^{(\ell, m)}$ denote the derivatives of $X_{k}(t, u)$, $Z_{k}(t, u)$ and $Y(t, u)$ evaluated at $t=u=0$. Therefore, the problem boils down to determining the deterministic equivalents for $X_{k}(t, u), Z_{k}(t, u)$ and $Y(t, u)$ and taking their derivatives at $t=u=0$. This approach has been pursued in [4] with the only difference that in [4] the large scale fading attenuation has not been included in the channel model. This means that some attention must be paid when applying the results from [4] to the problem at hand since the effect of the large scale fading cancels out only in $\left(\widehat{\mathbf{H}} \mathcal{B}^{-1} \widehat{\mathbf{H}}\right)^{k}$. Skipping the mathematical details for space limitations, it turns out that the asymptotic equivalents for $Y^{(\ell, m)}$ and $Z_{k}^{(\ell, m)}$ are in the same form of those computed in [4] once $\operatorname{tr}(\mathbf{P})$ is replaced with $\operatorname{tr}(\mathbf{P B})$. In doing so, the following lemma can be proved (to ease understanding the same notation of [4] is used):

Lemma 1. In the asymptotic regime, the following convergences hold true:

$$
X_{k}^{(\ell, m)}-\bar{X}^{(\ell, m)} \underset{M, K \rightarrow+\infty}{\stackrel{a . s}{\longrightarrow}} 0
$$

where $\bar{X}^{(\ell, m)}=a_{\ell} a_{m}$ with

$$
a_{\ell}=\sqrt{1-\tau^{2}} \sum_{k=0}^{\ell}\left(\begin{array}{l}
n \\
k
\end{array}\right) \delta^{(k)} f^{(\ell-k)}
$$

while the expressions of $\delta^{(k)}$ and $f^{(\ell-k)}$ are provided in [4]. Moreover, we also have:

$$
\begin{gathered}
-K p_{k} X_{k}^{(\ell, m)}+Z_{k}^{(\ell, m)}-\operatorname{tr}(\mathbf{P B}) b^{(\ell, m)} \frac{a . s}{M, K \rightarrow+\infty} 0 \\
Y^{(\ell, m)}-\operatorname{tr}(\mathbf{P B}) c^{(\ell, m)} \frac{a . s}{M, K \rightarrow+\infty} 0
\end{gathered}
$$

where the expressions of $b^{(\ell, m)}$ and $c^{(\ell, m)}$ take the same values as those derived in [4].

Let $\mathbf{a}$ be the $J \times 1$ vector defined as $[\mathbf{a}]_{\ell}=a_{\ell}$ and call $\mathbf{B}$ and $\mathbf{C}$ the $J \times J$ matrices whose elements are given by $[\mathbf{B}]_{\ell, m}=b^{(\ell, m)}$ and $[\mathbf{C}]_{\ell, m}=c^{(\ell, m)}$. Then, the following result is obtained:

Corollary 1. For any $k$ :

$$
\left(\left\|\mathbf{A}_{k}-\overline{\mathbf{A}}_{k}\right\|,\left\|\mathbf{B}_{k}-\overline{\mathbf{B}}_{k}\right\|\right) \underset{M, K \rightarrow+\infty}{\stackrel{a . s}{\longrightarrow}} 0
$$

and

$$
\|\mathbf{E}-\overline{\mathbf{E}}\| \underset{M, K \rightarrow+\infty}{\stackrel{a . s}{\longrightarrow}} 0
$$

where:

$$
\left[\overline{\mathbf{A}}_{k}\right]_{\ell, m}=\beta_{k}^{2} \mathbf{a a}^{T}, \quad\left[\overline{\mathbf{B}}_{k}\right]_{k}=\beta_{k} \operatorname{tr}(\mathbf{P B}) \mathbf{B}
$$

and

$$
[\overline{\mathbf{E}}]=\operatorname{tr}(\mathbf{P B}) \mathbf{C}
$$

\section{AsYMPTOTIC OPTIMIZATION OF TPE PRECODING}

The asymptotic analysis above is used in the sequel to compute the weighting vector $\mathbf{w}$ in (6) that minimizes the transmit power while satisfying SINR constraints. Mathematically, this amounts to solving the following optimization problem:

$$
\begin{aligned}
\mathcal{P}_{1}: \quad \min _{\mathbf{w}, \mathbf{p}} & \operatorname{tr}(\mathbf{P} \mathcal{B}) \mathbf{w}^{\mathrm{T}} \mathbf{C w} \\
\text { subject to } & \mathbf{w}^{\mathrm{T}} \mathbf{F}_{k} \mathbf{w} \geq \frac{\gamma_{k}}{\beta_{k}} \sigma^{2} \quad k=1, \ldots, K
\end{aligned}
$$

where $\mathbf{F}_{k}=K p_{k} \beta_{k} \mathbf{a a}^{\mathrm{T}}-\gamma_{k} \operatorname{tr}(\mathbf{P B}) \mathbf{B}$. Note that not all vectors $\gamma=\left[\gamma_{1}, \cdots, \gamma_{K}\right]$ are feasible. Indeed, a necessary and sufficient condition can be established using the same approach as in [14]. To this end, note that the constraints can be written in matrix form as:

$$
(\mathbf{I}-\mathbf{D}(\boldsymbol{\gamma}) \mathbf{G}) \mathbf{p} \geq \mathbf{v}
$$


where $\geq$ stands for the element-wise comparison of vectors and $\mathbf{p}=$ $\left[p_{1}, \cdots, p_{K}\right]^{\mathrm{T}}, \mathbf{D}(\gamma)=\operatorname{diag}\left(\gamma_{1}, \cdots, \gamma_{K}\right)$ whereas

$$
\begin{aligned}
{[\mathbf{v}]_{k} } & =\frac{\gamma_{k} \sigma^{2}}{K \beta_{k}^{2} \mathbf{w}^{\mathrm{T}} \mathbf{a}^{\mathrm{T}} \mathbf{w}}, \\
{[\mathbf{G}]_{i, j} } & =\frac{\beta_{j} \mathbf{w}^{\mathrm{T}} \mathbf{B} \mathbf{w}}{K \beta_{i} \mathbf{w}^{\mathrm{T}} \mathbf{a} \mathbf{a}^{\mathrm{T}} \mathbf{w}} .
\end{aligned}
$$

From Lemma 1 of [14], the following necessary and sufficient condition can be derived:

Lemma 2. A set $\gamma$ is feasible if and only if there exists a vector $\mathbf{w}$ satisfying

$$
r(\mathbf{G D}(\gamma))<1
$$

where $r(\cdot)$ computes the spectral radius of the enclosed matrix.

Based on the above lemma, the following result is established.

Corollary 2. A vector $\gamma=\left[\gamma_{1}, \gamma_{2}, \ldots, \gamma_{K}\right]^{T}$ is feasible if and only if

$$
\underline{\gamma}=\frac{1}{K} \sum_{k=1}^{K} \gamma_{k}<\mathbf{a}^{T} \mathbf{B}^{-1} \mathbf{a} .
$$

Proof: Since matrix $\mathbf{G}$ is non-negative with rank 1, $r(\mathbf{G D}(\boldsymbol{\gamma}))=\operatorname{tr}(\mathbf{G D}(\boldsymbol{\gamma}))$. The vector $\boldsymbol{\gamma}$ is feasible if and only if there exists $\mathbf{w} \in \mathbb{R}^{J \times 1}$ such that:

$$
\frac{\mathbf{w}^{\mathrm{T}} \mathbf{B} \mathbf{w}}{\mathbf{w}^{\mathrm{T}} \mathbf{a} \mathbf{a}^{\mathrm{T}} \mathbf{w}} \frac{1}{K} \sum_{i=1}^{K} \gamma_{k}<1
$$

or equivalently:

$$
\max _{\mathbf{w}} \frac{\mathbf{w}^{\mathrm{T}} \mathbf{a} \mathbf{a}^{\mathrm{T}} \mathbf{w}}{\mathbf{w}^{\mathrm{T}} \mathbf{B} \mathbf{w}}>\underline{\gamma} .
$$

The necessary and sufficient condition follows by noticing that $\max _{\mathbf{w}} \frac{\mathbf{w}^{\mathrm{T}} \mathbf{a}^{\mathrm{T}} \mathbf{w}}{\mathbf{w}^{\mathrm{T}} \mathbf{B} \mathbf{w}}=\mathbf{a}^{\mathrm{T}} \mathbf{B}^{-1} \mathbf{a}$.

Next, we assume that the feasibility condition in Corollary 2 is always satisfied. In these circumstances, the optimal power vector $\mathbf{p}^{\star}$ is such that the SINR constraints are satisfied with equality. From (17), we obtain:

$$
\mathbf{p}^{\star}=\left(\mathbf{I}_{K}-\mathbf{D}(\gamma) \mathbf{G}\right)^{-1} \mathbf{D}(\gamma) \mathbf{v}
$$

The objective function of $\mathcal{P}_{1}$ is thus given by:

$$
\operatorname{tr}\left(\boldsymbol{B} \operatorname{diag}\left((\mathbf{I}-\mathbf{D}(\boldsymbol{\gamma}) \mathbf{G})^{-1} \mathbf{v}\right)\right) \mathbf{w}^{\mathrm{T}} \mathbf{C w}
$$

Recalling the definitions of $\mathbf{v}$ and $\mathbf{G}$ given above it follows that when the constraints are satisfied with equality, the objective function of $\mathcal{P}_{1}$ becomes irrespective to the norm of $\mathbf{w}$. This means that solving $\mathcal{P}_{1}$ requires to determine the optimal direction of $\mathbf{w}$. However, this is a challenging task since $\mathcal{P}_{1}$ is not convex. To overcome this problem, we sum the constraints and perform the optimization with respect to $\mathbf{u}=\sqrt{\operatorname{tr}\left(\mathbf{P}^{\star} \mathcal{B}\right)} \mathbf{w}$. In doing so, we obtain

$$
\begin{aligned}
\mathcal{P}_{2}: \quad \min _{\mathbf{u}} & \mathbf{u}^{T} \mathbf{E u} \\
\text { subject to } & \mathbf{u}^{T} \mathbf{a a}^{\mathrm{T}} \mathbf{u}-\underline{\gamma} \mathbf{u}^{T} \mathbf{B u} \geq \kappa \sigma^{2}
\end{aligned}
$$

with $\kappa$ given by

$$
\kappa=\frac{1}{K} \sum_{k}^{K} \frac{\gamma_{k}}{\beta_{k}} .
$$

As the optimization of $\mathcal{P}_{2}$ is performed on a larger set, the optimal solution of $\mathcal{P}_{2}$ is the same as that of $\mathcal{P}_{1}$ since we assume the feasibility condition to be satisfied. Moreover, one can further work out the constraint of $\mathcal{P}_{2}$ to make it convex. To this end, note that one

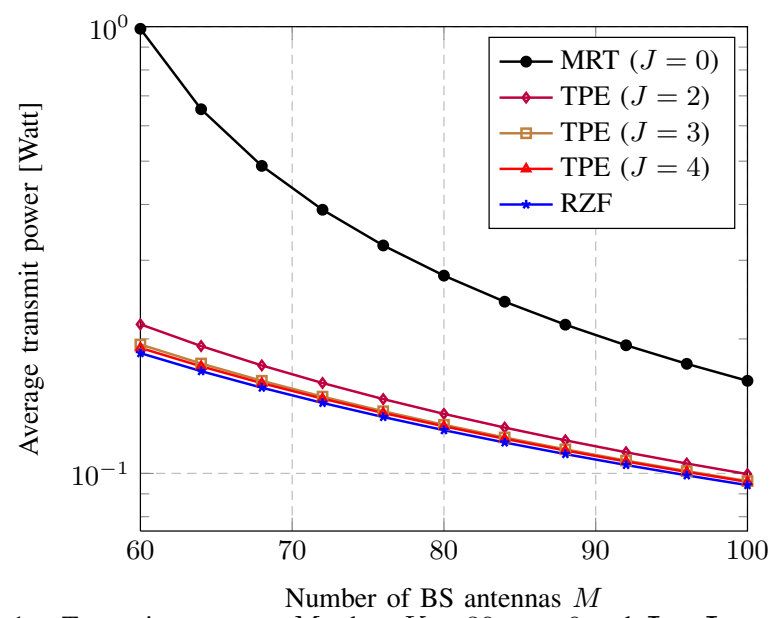

Fig. 1. Transmit power vs. $M$ when $K=30, \tau=0$ and $\mathbf{\Phi}=\mathbf{I}_{M}$.

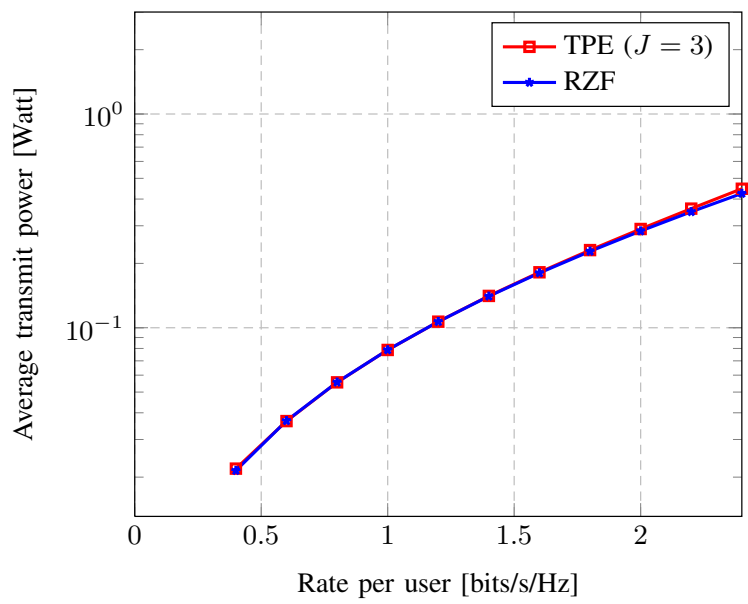

Fig. 2. Transmit power vs. rate per user when $K=30, M=60, \tau=0$ and $\boldsymbol{\Phi}=\mathbf{I}_{M}$.

can assume (without loss of generality) that the optimal vector $\mathbf{u}^{*}$ satisfies $\mathbf{a}^{\mathrm{T}} \mathbf{u}^{\star} \geq 0$, thereby leading to the following convex equivalent constraint:

$$
\mathbf{a}^{\mathrm{T}} \mathbf{u} \geq \sqrt{\underline{\gamma} \mathbf{u}^{T} \mathbf{B u}+\kappa \sigma^{2}}
$$

With this new constraint at hand, the lagrangian corresponding to $\mathcal{P}_{2}$ takes the form:

$$
\mathcal{L}(\mathbf{u}, \lambda)=\mathbf{u}^{T} \mathbf{E} \mathbf{u}+\lambda\left(\underline{\gamma}^{T} \mathbf{B u}+\kappa \sigma^{2}-\left(\mathbf{a}^{\mathrm{T}} \mathbf{u}\right)^{2}\right)
$$

with $\lambda>0$. From the Karush-Kuhn-Tucker conditions, we know that the optimal weight vector and the optimal Lagrange multiplier satisfy $\mathbf{E} \mathbf{u}^{\star}+\lambda^{\star} \underline{\gamma} \mathbf{B} \mathbf{u}^{\star}=\lambda \mathbf{a} \mathbf{a}^{\mathrm{T}} \mathbf{u}^{\star}$ from which one gets

$$
\mathbf{u}^{\star}=\left(\mathbf{E}+\lambda^{\star} \underline{\gamma} \mathbf{B}\right)^{-1} \lambda^{\star} \mathbf{a a}^{\mathrm{T}} \mathbf{u}^{\star}
$$

and

$$
\lambda^{\star}=\frac{1}{\mathbf{a}^{\mathrm{T}}\left(\mathbf{E}+\lambda^{\star} \underline{\gamma} \mathbf{B}\right)^{-1} \mathbf{a}} .
$$

In summary, the optimal $\mathbf{u}^{\star}$ is found to be collinear with the vector $\left(\mathbf{E}+\lambda^{\star} \underline{\gamma} \mathbf{B}\right)^{-1}$ a whereas the Lagrange coefficient $\lambda^{\star}$ is the unique positive solution of 27.

\section{NUMERICAL RESULTS}

Numerical results are now used to assess the performance of the proposed TPE precoding technique. We assume that the UEs are uniformly distributed in the coverage area, which is assumed to be 


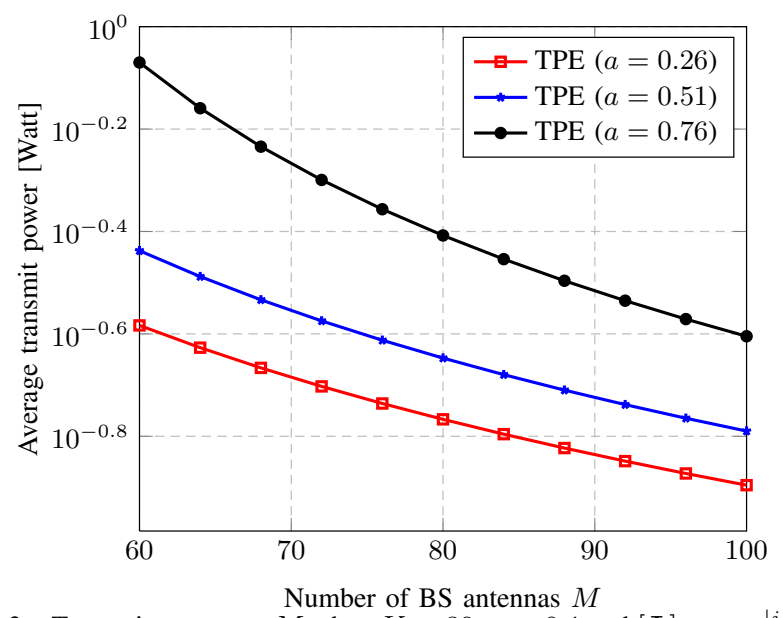

Fig. 3. Transmit power vs. $M$ when $K=30, \tau=0.1$ and $[\boldsymbol{\Phi}]_{i, j}=a^{|i-j|}$.

circular with radius $\mathrm{D}=250 \mathrm{~m}$ and minimum distance $\mathrm{D}_{\min }=$ $35 \mathrm{~m}$. The large scale fading is dominated by the path-loss and is modeled as $\beta_{k}=d_{0} / d_{k}^{\eta}$ where $d_{k} \geq \mathrm{D}_{\min }$ and $\eta \geq 2$ is the path-loss exponent whereas the constant $d_{0}$ regulates the channel attenuation at distance $\mathrm{D}_{\min }$. We set $\eta=3.76$ and $d_{0}=10^{-3.53}$. Moreover, the transmission bandwidth is fixed to $W=20 \mathrm{MHz}$ and the total noise power $W \sigma^{2}$ is $-97.8 \mathrm{dBm}$.

We begin by investigating the performance of TPE for different values of $J$. As a benchmark, we use the RZF precoding scheme illustrated in [6], which is shown to be optimal when the channel covariance matrix $\boldsymbol{\Phi}=$ is set to $\mathbf{I}_{M}$ and perfect CSI is available. Fig. 1 plots the average transmit power in Watt vs. $M$ when $K=30$ and the SINR constraints $\gamma_{k}$ are set equal to $\gamma_{k}=2_{k}^{r}-1$ with $r_{k}$ being randomly taken from the interval $[1,2] \mathrm{bit} / \mathrm{s} / \mathrm{Hz}$. The RZF precoding and the TPE precoding guarantee almost the same performance even for small values of $J$ and the gap of performance is decreasing when $M$ grows large. Observe that $J=0$ corresponds to the maximum ratio transmit (MRT) precoding technique. Fig. 2 plots the average transmit power when $J=3$ and the same rate $r$ must be satisfied to each UE. This amounts to setting $\gamma_{k}=\gamma=2^{r}-1$ for $k=$ $1, \ldots, K$. In particular, we assume that $r$ spans the interval from 0.4 to $2.4 \mathrm{bit} / \mathrm{s} / \mathrm{Hz}$. As seen, TPE requires the same power as RZF for all investigated values of rates. This is however achieved with a reduced complexity as illustrated in [4].

We proceed investigating the performance of the TPE precoding technique when $\tau=0.1$ and $[\boldsymbol{\Phi}]_{i, j}=a^{|i-j|}$. Fig. 3 reports the average transmit power as a function of $M$ for different values of $a$ when $J=3$. As seen, the average transmit power increases as the correlation coefficient $a$ becomes larger. This is due the fact that increasing $a$ reduces the degrees of freedom of the channel and consequently forces the BS to use more power for serving the UEs. Fig 4 investigates the impact of the correlation factor $a$ on the feasibility of the SNR constraints. Similar to Fig. 2, we consider the case in which the same rate $r$ must be satisfied for all UE, i.e., $\gamma_{k}=2^{r}-1$, and plot the average transmit power for $r$ spanning the interval from 0.4 to $8 \mathrm{bit} / \mathrm{s} / \mathrm{Hz}$. As expected, increasing the correlation factor reduces the feasibility region of the SNR constraints.

\section{CONCLUSIONS}

In this work, we have focused on the design of a linear TPE precoding scheme to minimize the power consumption while satisfying SINR constraints under the assumption of imperfect CSI. The design has been conducted in the asymptotic regime in which $M, K \rightarrow \infty$ with the same pace. In particular, we have determined

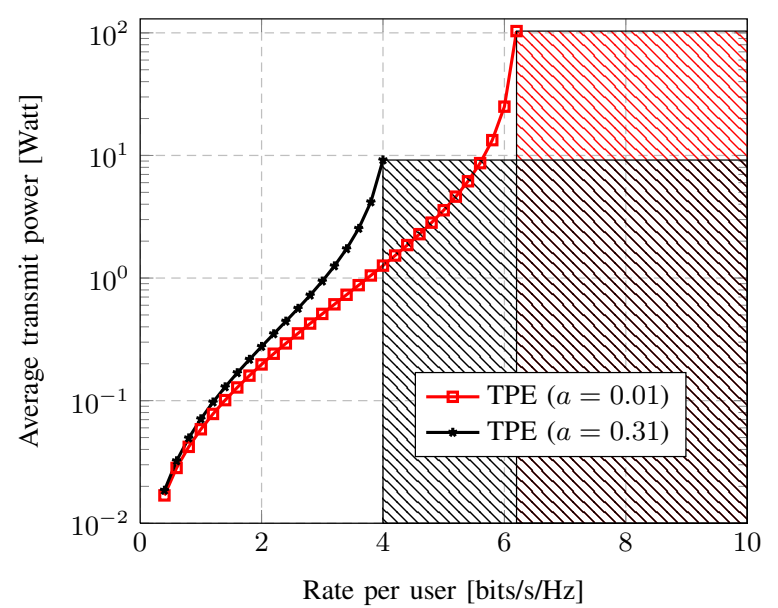

Fig. 4. Transmit power vs. rate per user when $K=30, M=60, \tau=0.1$ and $[\boldsymbol{\Phi}]_{i, j}=a^{|i-j|}, J=3$.

the optimal weights that minimize the total asymptotic transmit power while meeting a set of target SINR requirements. Comparisons have been made with the optimal RZF precoding technique under the assumption of perfect CSI. While exhibiting a lower complexity, the TPE scheme has been shown to achieve almost the same performance of the optimal RZF precoding. Numerical results have been also used to evaluate the impact of imperfect CSI and of the correlation among BS antennas.

\section{REFERENCES}

[1] E. G. Larsson, O. Edfors, F. Tufvesson, and T. L. Marzetta, "Massive MIMO for next generation wireless systems," IEEE Commun. Mag., vol. 52, no. 2, pp. 186-195, Feb. 2014.

[2] S. Wagner, R. Couillet, M. Debbah, and D. T. M. Slock, "Large system analysis of linear precoding in MISO broadcast channels with limited feedback," IEEE Trans. Inf. Theory, vol. 58, no. 7, pp. 4509-4537, July 2012.

[3] J. Hoydis, S. ten Brink, and M. Debbah, "Massive MIMO in the UL/DL of cellular networks: How many antennas do we need?," IEEE J. Sel. Areas Commun., vol. 31, no. 2, pp. 160-171, February 2013.

[4] A. Müller, A. Kammoun, E. Björnson, and M. Debbah, "Linear precoding based on polynomial expansion: Reducing complexity in massive MIMO (extended version)," IEEE Trans. Signal Process., Apr. 2014, Submitted, arXiv:1310.1806.

[5] A. Kammoun, A. Müller, E. Björnson, and M. Debbah, "Linear precoding based on polynomial expansion: Large-scale multi-cell MIMO systems,” IEEE J. Sel. Topics Signal Process., 2014.

[6] L. Sanguinetti, E. Björnson, M. Debbah, and A. Moustakas, "Optimal linear precoding in multi-user MIMO systems: A large system analysis," in Proceedings of Globecom 2014, Austin, Texas, 2014.

[7] E. Björnson, L. Sanguinetti, J. Hoydis, and M. Debbah, "Optimal design of energy-efficient multi-user MIMO systems: Is massive MIMO the answer?," submitted to IEEE Trans. Wireless Comm., April 2014.

[8] L. Sanguinetti, A. L. Moustakas, E. Björnson, and M. Debbah, "Large system analysis of the energy consumption distribution in multi-user MIMO systems with mobility," submitted to IEEE Trans. Wireless Comm., May 2014.

[9] A. Wiesel, Y.C. Eldar, and S. Shamai, "Linear precoding via conic optimization for fixed MIMO receivers," IEEE Trans. Signal Process., vol. 54, no. 1, pp. 161-176, Jan. 2006.

[10] N. Shariati, E. Björnson, M. Bengtsson, and M. Debbah, "Lowcomplexity channel estimation in large-scale MIMO using polynomial expansion," in Proc. IEEE Int. Symp. Personal, Indoor and Mobile Radio Commun. (PIMRC), 2013.

[11] J. Hoydis, M. Debbah, and M. Kobayashi, "Asymptotic moments for interference mitigation in correlated fading channels," in Proc. Int. Symp. Inf. Theory (ISIT), 2011.

[12] S. Moshavi, E.G. Kanterakis, and D.L. Schilling, "Multistage linear receivers for DS-CDMA systems," Int. J. Wireless Information Networks, vol. 3, no. 1, pp. 1-17, Jan. 1996. 
[13] W. Hachem, O. Khorunzhy, P. Loubaton, J. Najim, and L. A. Pastur, "A new approach for capacity analysis of large dimensional multi-antenna channels," IEEE Trans. Inf. Theory, vol. 54, no. 9, pp. 3987-4004, Sept. 2008.

[14] P. Hande, S. Rangan, M. Chiang, and X. Wu, "Distributed uplink power control for optimal SIR assignment in cellular data networks," IEEE Trans. Ind. Informat., vol. 14, April 2006. 